\title{
La torre de Toya: consideraciones acerca de su cronología
}

Irene Montilla Torres.

Sonia Pérez Alvarado *

\section{INTRODUCCIÓN}

La zona objeto de nuestro estudio pertenece a la Comarca del Guadiana Menor. Se trata de un territorio centrado en la vega del río Toya, afluente del Guadiana Menor, y algunos de sus tributarios: el Barranco del Castillo, la Rambla del Piojo, etc. que la convierten en uno de los puntos de acceso a la Sierra de Segura-Cazorla. El sur de la vega está limitada por la Serranía de Toya, con unas altitudes medias que oscilan entre los $550 \mathrm{~m}$, en el Cerro del Castillo, y los $420 \mathrm{~m}$ de la vega del río.

El lugar, situado a $6 \mathrm{~km}$ de la población de Peal de Becerro (Jaén), corresponde al emplazamiento de la antigua ciudad ibero-romana de Tugia, aunque la secuencia histórica del asentamiento comienza en la edad del cobre, llegando a época almohade. En el punto más elevado se localiza la Torre de Toya, objeto de este estudio!

La ciudad se ubicaba presumiblemente en la ladera noroeste del cerro, donde aparecen dispersos numerosos fragmentos de "terra sigillata" itálica, gálica e hispánica que nos dan una cronología del siglo I. Plinio y Ptolomeo le adjudican el estatus de municipio flavio. En el siglo Il la mayoría de las ciudades experimentaron una fuerte crisis que motivó su abandono con el consiguiente proceso de ruralización (CHOCLÁN, CASTRO, 1988; ABASCAL, ESPINOSA, 1989). A pesar de ello, parece que Tugia siguió ampliamente poblada, como lo demuestra la presencia de "sigillatas" paleocristianas que pueden fecharse hacia mediados del siglo $\mathrm{V}$, y que aparecen en la misma zona que las anteriores. El empleo de materiales reutilizados en la torre, como luego veremos, indica que la construcción de ésta tuvo que ser posterior al auge de la ciudad, muy posiblemente después del siglo $\mathrm{V}$.

\section{LA TORRE DE TOYA. CARACTERÍSTICAS FORMALES}

La torre de Toya (Lám. I) presenta una planta cuadrada de II metros de lado. Su alzado muestra dos cuerpos claramente diferenciados. El primero está formado por una estructura de piedras irregulares unidas por argamasa, aparentemente rica en cal, forrada al exterior por sillares de época romana reutilizados. Muestra de ello es el empleo de una inscripción (Lám. 2), una quicialera, un sillar almohadillado aislado, etc. El aparejo de los mismos es a soga y tizón trabando éste con la argamasa, lo que proporciona gran solidez a la estructura. Los sillares aparecen enripiados con piedras pequeñas, argamasa y cerámica. En el muro este se encuentra una cruz griega grabada en uno de los sillares que pensamos sería la marca de un taller o de un artesano. En el interior, se utiliza el sillarejo con un aparejo a soga que presenta el mismo tipo de argamasa que en el exterior. La

* Área de Historia Medieval. Departamento de Territorio y Patrimonio Histórico. Universidad de Jaén.

I Nuestro más sincero agradecimiento a D. Juan R. Montilla Torres y D. francisco Martínez Quirce. 
altura de este primer cuerpo varía en función de la pendiente del cerro, oscilando entre los $2.30 \mathrm{~m}$ de la cara norte y los $4.45 \mathrm{~m}$ de la sur.

En el segundo cuerpo, el material constructivo empleado es radicalmente distinto pasándose de los sillares al tapial, del que aún se conservan hasta cuatro cajones completos de unos $80 \mathrm{~cm}$ cada uno.

De este modo, la torre constaría de, al menos, dos plantas y quizá una terraza, como se puede obsevar por las huellas de posibles vigas. Suponemos que el acceso a las distintas plantas se resolvería mediante una escalera de la que no hemos encontrado resto alguno, quizás por ser de madera.

En cuanto a la puerta de acceso a la torre, sólo cabe situarla en el muro norte puesto que es el único que presenta un gran vano. Además, ésta es la cara más destrozada, con diferencia, sobre las otras, siendo también la parte más accesible. Se trataría de una puerta en altura, es decir, situada en el tramo medio, puesto que no aparecen quicialeras ni ningún otro elemento consustancial a la misma.

El interior se divide en dos espacios por un muro medianero con dirección norte-sur de unos $90 \mathrm{~cm}$ de ancho. En la zona que queda al este se construye un aljibe de tapial de considerables dimensiones, al tiempo que se rellena la otra, también con tapial, con el fin de darle una mayor consistencia al aljibe, impidiendo así su fragmentación. Podemos suponer, en función del material constructivo utilizado, que tanto esta construcción de tapial como el segundo cuerpo de la torre, serían coetáneos.

Posteriormente, la torre sufre otra modificación con la construcción de una nueva bóveda en el aljibe, de la que se conserva su arranque (Lám. 3). Los sillares presentan una argamasa de color rojizo rica en yeso.

En el exterior de la torre y aprovechando la orografía del terreno, se podía apreciar un lienzo (Lám. 4) que con toda probabilidad formaría parte de una muralla (destruida en la actualidad). Estaba construida a base de mam- postería trabada con la misma argamasa rojiza que encontramos en el aljibe. Los restos se situaban en la ladera noroeste $y$, por su dirección, parece que cerrarían el perímetro que ocupa la meseta inmediata a la torre. Constaba de varias torres macizas, situadas a una distancia de I I m aproximadamente, así como de un acceso en pasillo recto que no presentaba ninguna complejidad aparente en su estructura. Probablemente se levantaba sobre los restos de otra muralla o elemento defensivo de época anterior, puesto que debajo de la misma encontramos una hilada de piedras sin argamasa, de la que desconocemos su cronología.

Aunque pudiera pensarse que la torre responde a un único periodo histórico debido al enrase del primer cuerpo, toda una serie de cuestiones $y$, fundamentalmente, el empleo de distintos materiales constructivos, nos inducen a pensar que estamos ante, al menos, dos momentos cronológicos distintos como estudiaremos a continuación.

\section{CRONOLOGÍA}

\section{Época visigoda.}

Resulta extremadamente difícil determinar las características del núcleo de población que en estas fechas existía en la zona, ya que la cerámica de los siglos $\mathrm{VI}$ y $\mathrm{VII}$ encontrada es muy escasa, si bien hay que tener en cuenta que los estudios cerámicos de este periodo no están muy desarrollados aún. Por lo general, la escasez de materiales suele interpretarse como resultado de una reducción de la población que habita en el lugar del que se trate.

No debemos olvidar que, según diversos autores (CHOCLÁN, CASTRO, 1988; SALVATIERRA, 1992; ACIÉN, 1995 , etc.), en este periodo se produce la crisis generalizada de la ciudad romana que conlleva la desarticulación del sistema que la reproducía socialmente. Así, en los casos de Cástulo, Isturgi, lliturgi, etc. se aprecia claramente una reducción del perímetro urbano en relación al existente en la época de su apogeo. Sin embargo, esta afirmación debe matizarse en función de las zonas que se analicen. Buena 
muestra de ello son los resultados obtenidos a partir de las excavaciones arqueológicas en curso en la ciudad visigoda de Recópolis, fundada por Leoviglido, y en el Tolmo de Minateda (Hellín, Albacete). En este último caso, se puede observar su "regeneración" durante este periodo como nudo de comunicaciones entre bizantinos y visigodos (OLMO, 1992; GUTIÉRREZ, 1996).

Por su parte, las fuentes escritas visigodas siguen calificando ciertos núcleos de población como ciudades, lo que ha llevado a algunos autores a defender la permanencia de las mismas (SALVADOR, 1990). Sin embargo, en la mayoría de los casos se trata de sedes episcopales que no cumplen las funciones que se consideran mínimas para hablar de ciudad, es decir, presencia de un mercado, existencia de un artesanado, así como de unos órganos administrativos que la articulen (TORRES BALBÁS, 197I).

De este modo, y en función del escaso material arqueológico fechable en este periodo hallado en nuestra prospección, es razonable suponer que la antigua Tugia no es ajena al proceso que acabamos de describir. Posiblemente había entrado en un periodo de decadencia y de reducción importante de su población, pero es necesario situar ésta en el contexto concreto de la zona.

A partir del siglo $V$ se producen grandes cambios en Occidente, incluida la Península lbérica, cuando el Imperio Romano es desbordado y ocupado por los invasores germanos, que crean a su vez varios reinos sobre los territorios de las antiguas provincias. En concreto, en la Península, tras casi medio siglo de vicisitudes se harán con el poder los visigodos, estableciendo su capital en Toledo, y tratando de dominar todo el conjunto peninsular, aunque parece que hasta Leovigildo el valle del Guadalquivir escapará a su control.

Es en este marco donde hay que situar la invasión bizantina de la Península en el 55।, ocupando una franja comprendida entre el cabo de la Nao y Gibraltar que se adentraba en tierras de la Bética y la Cartaginense y que recibirá el nombre de Spania. Será Leovigildo (569603) quien intente consolidar unas fronteras seguras con los bizantinos, empujándolos hacia la costa y alejándolos del rico valle del Guadalquivir que pretendía controlar. De este modo, lanzará una gran ofensiva entre el 570 y 57I que culminará con la conquista de Basti, Acci y Asidona (GARCíA, 1991).

Dada su situación, hay dos momentos en que Tuia pudo pasar a dominio visigodo. $\bigcirc$ fue controlada por Leovigildo antes del ataque a Baza o lo hizo durante la misma campaña, puesto que es improbable que dejase una localidad de aparente importancia en manos enemigas en su retaguardia (SALVATIERRA, 1996).

Sin embargo, el territorio al que la misma da acceso, la Sierra de Segura-Cazorla que, según las fuentes visigodas parece corresponder a la Orospeda, fue la zona del Alto Guadalquivir que resistió más tiempo al dominio visigodo. Amparados en las dificultades del terreno, sus habitantes permanecieron independientes hasta el 577, momento en que Leoviglido lanzó una campaña específica contra ellos, en el curso de la cual conquistó, según las fuentes visigodas, ciudades y castillos.

No obstante, Thompson considera que la zona dependía en alguna forma de las grandes poblaciones del valle del Guadalquivir en manos de la aristocracia hispano-romana, y que se habría hecho independiente tras la conquista de Córdoba por los visigodos en el 572, constituyendo un peligro potencial para la estabilidad del área recién conquistada por Leovigildo (THOMPSON, 1969). Es posible que en este marco de continuas luchas y con la cercanía del "limes" bizantino se construyese la torre de Toya.

Cabría pensar que nos encontramos frente a una obra encargada por el Estado visigodo y ejecutada por un ingeniero que conocía las reglas de la poliorcética, ya que se trata de una obra de relativa envergadura. De este modo, la torre se constituiría o bien como elemento para la defensa de la población frente a los bizantinos o bien para el control de la misma, puesto que no domina ningún paso sino el valle sobre el que se asienta la ciudad. Esta construcción, por tanto, formaría parte de la 
política que los visigodos desarrollarán contra los bizantinos con posterioridad a Leovigildo.

Otro elemento de la misma sería la aplicación a este lugar de la ley antijudía de Sisebuto (612-62I), publicada en los primeros meses de su reinado y en la que aparecen una serie de territoria con el nombre de ciudades, en las que se encontrarían comunidades judías lo suficientemente significativas como para que el monarca godo les dedicase una ley específica. Están distribuidas a lo largo del valle medio y alto del Guadalquivir. Se trata de Aurgi, Barbi, Beatia, Egabrum, Epagrum, lliturgi, Sturgi, Tuia, Tutugi.

Se encarga su ejecución a las dos instituciones más importantes del reino visigodo: Estado e Iglesia, implicando específicamente a los tres obispos de la zona: Agapio de Córdoba, Cecilio de Mentesa y Agapio de Tucci, y a los iudices de los nueve territoria citados. Además, la ley debía ser cumplida antes del uno de julio del 62I (SALVADOR, 1990; GARCíA, 199 I). F. Salvador ha señalado a este respecto la irregularidad que supone el hecho de que no se mencione al obispo de Egabrum (SALVADOR 1990). La única explicación plausible es que dicha sede estuviese vacante en esos momentos.

Debemos tener en cuenta que, aunque por lo general se coincide en que la reiteración de leyes es un claro indicio de que las mismas no se cumplían a plena satisfacción del poder que las dictaba, y que ello es de aplicación a la legislación antijudía (los hebreos seguían manteniendo grandes valedores entre los hispanoromanos e incluso entre la aristocracia cristiana y los obispos), no puede descartarse que ésta en concreto tuviera una mayor eficacia ya que es la única que se aplica a lugares específicos y cuya puesta en práctica se encarga a personajes concretos a los que se hace responsables antes de una fecha preestablecida. Además, según García Moreno (1991), la nueva legislación intentaría atacar a los sectores más ricos e influyentes de la comunidad judía, que con sus dádivas y sobornos debían de haber burlado las legislaciones anteriores.

Si observamos en el mapa (Fig. I) la situación de los lugares recogidos en la ley, parece que pueden diferenciarse dos grupos. El primero estaría formado por Tutugi, Tuia, Beatia, Aurgi, Sturgi e lliturgi, que tendrían el Guadalquivir como eje de comunicación, y el segundo agruparía a Epagrum, Egabrum y Barbi, a través del Guadalhorce y el Genil.

Debemos tener en cuenta que el comercio se encontraba en gran medida en manos de las comunidades judías (AGUIRRE, SALVATIERRA, 1989), en parte por pertenecer a las colonias de mercaderes situadas en las costas meridionales, y en parte porque la continua legislación en su contra les reducía los campos económicos en los que desenvolverse (SALVADOR, 1990).

En esta situación, podríamos suponer la existencia de un intercambio de productos desde Carthago Spartaria, donde además existía una importante comunidad judía, con las ciudades que conformarían el primer grupo (Tutugi, Tuia, Beatia, Aurgi, lliturgi, Sturgi) con el eje Guadiana Menor-Guadalquivir como ruta básica; y desde Malaca con Barbi, Egabrum y Epagrum, con el eje Guadalhorce-Genil. De este modo, con la ley antijudía se impide la continuación de las redes comerciales con la zona bizantina, intentando ahogar esta última (como hipótesis puede suponerse que los bizantinos estarían interesados en recibir sobre todo productos de consumo, entregando a cambio objetos de lujo), al tiempo que se les asestaba un golpe decisivo a las comunidades judías, que serían las menos interesadas en la expulsión de los bizantinos por motivos obvios y de las que quizás se temía una actitud favorable hacia los mismos.

Hay que tener en cuenta que esta ley precede en pocos meses a la conquista, por Sisebuto, de Malaca y Sidona, a partir de las bases estratégicas del Alto Guadalquivir, con lo que la provincia imperial debió de quedar reducida a una limitada franja territorial en torno a la importante plaza fuerte de Carthago Spartaria (GARCíA, 1991).

Por otra parte, el acoso a las comunidades hebreas podría considerarse como el punto final del proceso de desintegración de la ciudad, iniciado en el siglo II. 
En función del material cerámico encontrado, junto a la mención de Tuia en las fuentes visigodas; de la situación estratégica de la zona con respecto al control del limes bizantino -sobre todo si tenemos en cuenta el carácter fluctuante intrínseco a todo limes; de las revueltas campesinas de la zona; $y$, fundamentalmente, del aparejo utilizado en su construcción que no es característico de construcciones romanas ni islámicas y del que sólo conocemos un paralelo en la Península, concretamente la torre de acceso del yacimiento del Tolmo de Minateda (Hellín, Albacete), fechada por sus excavadores en época visigoda, planteamos la posibilidad de su construcción por parte del Estado visigodo como ya hemos señalado anteriormente.

No obstante, existe una importante dificultad. Hay noticias de la existencia en la torre de una inscripción, desaparecida de su lugar al parecer ya en el siglo pasado, aunque ha sido citada repetidamente por distintos autores, empleando las primeras descripciones que se hicieron.

Se trata de una inscripción monumental con una única línea de texto ocupando su parte superior y dos grandes discos con una estrella de seis puntas cada uno, en la parte inferior (GONZÁLEZ, MANGAS, 1991).

El primero en mencionarla es F. Salvador Laín y Rojas en carta con fecha de 24 de septiembre de 1818 y dirigida al, por aquel entonces, Director de la Real Academia de la Historia, D. Francisco Martínez Marina. Esta carta, junto con las observaciones realizadas a algunos de los datos recogidos en la misma, efectuadas en octubre del mismo año por D. José Antonio Conde, miembro también de la Academia, y las de otros autores posteriores como Hübner, fueron recopiladas en 1909 por el P. Fidel Fita.

Según Laín y Rojas, la inscripción se encontraba en el lado norte de la torre de Toya. El escaso texto lo interpretó como "FANTE LERAVIO" y lo tradujo "por haber hablado Leravio". Además pensaba que se trataba de una pieza muy antigua "y de los tiempos en que no estaban las Gentes de la tierra mui acostumbrados al Alphabeto Latino ó Romano" (FITA, 1909:470).
El propio J. A. Conde contradice al erudito local. Para este miembro de la Academia, el carácter de la inscripción es tal que "no puede hacerse juicio de su verdadera lectura, y me parece de tiempo Góttico" (FITA, 1909:476).

A. Hübner en su Inscriptiones Hispaniae Christianae, publicado en $|87|$, al igual que en el suplemento del mismo, editado en 1900, recoge la inscripción citando a Laín y Rojas. Señala que se encuentra una copia de la misma en el Museo Arqueológico de Madrid (actual Museo Arqueológico Nacional) y otra en el de Granada, realizadas por Manuel de Góngora. Al igual que Conde, plantea una cronología visigoda para la misma, fechándola en el siglo VIII y no en el $\mathrm{VI}$ como se ha deslizado en la recopilación de González y Mangas (199|). Al mismo tiempo, baraja la posibilidad, siguiendo a Méli$\mathrm{da}$, de que se tratase de un fragmento que continuaría en otra lápida. No parece convencerlo la lectura que realizó Gómez Moreno según la cual en la inscripción pondría "AD TE, LERARIO" y opta por AD(c)ELERARI O (bitum tuum ut celerius recipiare in caelum) (HÜBNER, I87| n० 173 y $\left.1900 n^{\circ} 402\right)$.

Fidel Fita recogerá en 1909 las interpretaciones de Laín, Conde y Hübner, haciendo una nueva revisión cuya conclusión, en cuanto a su cronología, seguirá la línea defendida por los dos últimos autores en el sentido de considerarla visigoda, pero señala que el tipo paleográfico que presenta la inscripción es claramente "visigótico del siglo VII", y cambia radicalmente la interpretación hecha hasta ese momento, ya que la identifica como el primer versículo del salmo I 22 "AD TE LEVAVI O(culos meos, qui habitas in caelis)" (FITA, 1909:476-477).

En 1962, J. Vives publicará un nuevo estudio de la misma. Su lectura sigue la línea de las anteriores aunque sólo cita a Hübner del que únicamente afirma que "no entendió nada" (1962: I 18). Vives la considera una inscripción monumental bien del coro, bien de otra parte de la iglesia, llegando a la conclusión de que su correcta lectura sería "+AD TE LEBAVI O(culos meos)" tratándose, por tanto, del "principio bien conocido del salmo I I2" (VIVES, 1962). 
Como vemos, las traducciones de Fita y Vives coinciden; ahora bien, el número del salmo indicado por Vives es distinto del que señaló Fidel Fita, que es quien lleva razón. Más recientemente, C. González y J. Mangas (199|) han elaborado una nueva compilación en la que siguen en todo a Vives. Aunque debemos señalar que la transcripción que recogen estos dos últimos autores y que atribuyen a Mommsen, es la misma que planteara Hübner, que no nombra a su compatriota en ningún momento.

Igualmente, hay discordancias en el término "levavi" que F. Fita escribe con $\vee$ en ambos casos y J. Vives con $B$ y $V$, lo que plantea un nuevo problema puesto que en latín este vocablo, que procede del infinitivo "levare", se escribe con V. Sin embargo, en epigrafía es frecuente la confusión entre $B$ y $\vee$ por lo que ambas transcripciones serían correctas².

De ser cierto que esta inscripción se encontraba situada realmente en la torre, y de confirmarse la cronología visigoda de la misma, de la que pensamos debería plantearse una nueva revisión por las dudas que aún presenta, tendríamos que desechar la hipótesis planteada acerca de la construcción de la torre en época visigoda siendo, evidentemente, posterior a la misma, como consideraremos más adelante.

Además de esta inscripción, J. Eslava (ESLA$\checkmark A$ y CEREZO 1989) menciona otra a la que otorgan una cronología árabe y que, según él, habría sido arrancada de la torre en 1935. Sin embargo, no hay ninguna otra noticia al respecto, y mucho menos sobre su posible contenido, época, etc.

\section{Época islámica.}

Las fuentes islámicas no nos dan noticia de Toya hasta al-Idrisi, geógrafo ceutí del siglo XII, que la califica como "hisn Tuya", aludiendo posiblemente tanto a la torre como a la muralla, que quizás en esos momentos ya la rodeaba.
Resulta difícil establecer lo sucedido entre los siglos VIII y XII. Pese a la falta de documentación, podemos plantear para el siglo VIII una continuidad con el poblamiento del último momento visigodo, ya que por un lado habría habido cierta permanencia de la población en el solar de la antigua ciudad visigoda de Tuia, que se extendía por las laderas norte y oeste del cerro y en su cumbre, así como por la presencia de otros núcleos menores.

A finales del siglo IX debemos mencionar las continuas sublevaciones muladíes que se están produciendo en todo el territorio de AlAndalus en respuesta al intento de centralización del emirato cordobés. En este sentido, observamos que el territorio de Toya no es ajeno a estas convulsiones, concretamente a las que se produjeron durante el emirato de 'Abd Allah (888-9/2), bajo el control de los hermanos Banu Habil. El mayor de ellos, Mundir, controló de forma efectiva las fortalezas que cabría situar entre la Sierra de Cazorla y la Depresión del Guadiana Menor ocupando las ya existentes e incluso construyendo otras. Dirigiendo al resto de sus hermanos, y siguiendo la conducta habitual entre la mayoría de los "encastillados", alternaron la lealtad con la rebeldía en su relación con el emir, según su conveniencia (AGUIRRE, JMÉNEZZ, 1979).

Es en este contexto donde debemos situar el problema cronológico de la torre. Hemos planteado ya los problemas que existen para defender una cronología visigoda. Por otro lado, tradicionalmente se ha venido manteniendo en la historiografía una cronología islámica bajomedieval (siglos XII-XIII), siendo J. Eslava el único que, muy recientemente, ha sugerido una cronología califal (ESLAVA y CEREZO 1989).

Por nuestra parte, no creemos que la primera fase pueda pertenecer a ninguno de esos momentos, ya que en época bajomedieval no se produce el reaprovechamiento de materiales romanos en la forma en que aquí sucede, y tenemos el cercano ejemplo de la torre de

\footnotetext{
2 Agradecemos la inestimable colaboración prestada por el Dr. D. Juan Higueras Maldonado en el esclarecimiento tanto del texto de Hübner como en la identificación del salmo.
} 
Sta. Eufemia en Cástulo, de construcción almohade, en la que no se reutilizan sillares pese a la abundancia y proximidad de los mismos. Una cronología de época omeya es bastante plausible si tenemos en cuenta algunas características técnicas, como el empleo de varios sillares seguidos dispuestos a tizón. No obstante, la propia irregularidad general del conjunto, y la reutilización de sillares romanos nos hace dudar de que se trate de una obra oficial, mucho menos califal, ya que los casos en que eso sucede son excepcionales.

Sin embargo, sí pudiera ser una construcción del siglo IX, una de esas fortificaciones realizadas por muladíes rebeldes y citadas por las fuentes. No debemos descartar la posibilidad de que los propios B. Habil llevarán a efecto la ejecución de la torre contando para ello con el material suficiente (todavía hoy puede encontrarse gran cantidad de sillares en la zona). Avalaría esta hipótesis:

I. Utilización de grandes sillares imitando así las grandes construcciones emirales, que parecen ser frecuentes desde 'Abd al-Rahman II.

2. Esto explicaría la presencia de las inscripciones latinas que ya hemos señalado. Tratándose supuestamente de una construcción muladí no sería extraño que quedaran al exterior, exhibiéndose de forma "propagandística" para manifestar la superioridad latina frente al dominio árabe. Aunque contrasta el hecho de que la única inscripción que aún permanece en su lugar, es de muy difícil lectura.

De este modo, la torre pudo ser construida por este grupo muladí con el fin de controlar y extorsionar a la población del entorno.

Tras el triunfo final del Estado cordobés con la instauración del Califato y con la consiguiente eliminación de todos los grupos rebeldes, la torre quedaría bajo dominio estatal para el control del entorno.

Al principio del dominio de almorávides y almohades se refiere la única cita que tenemos sobre Toya en época islámica. La cita, sin embargo, se limita a indicar la distancia existente desde la misma a Jódar y a Quesada, lo que parece sugerir que era una zona de paso (AGUIRRE, JIMENEZ 1979). El hecho de que se mencione revela que el lugar de Toya tenía cierta importancia, y ésta sólo parece posible relacionarla con la propia torre, construcción que inevitablemente debía de llamar la atención.

En esta época, los territorios de Al-Andalus, en general, y de Jaén, en particular, se caracterizarán por ir pasando de mano en mano complejizando enormemente el estudio de este periodo. Así, la situación política para la actual provincia de Jaén podría resumirse de la siguiente forma:

I 147-48: Alfonso VII conquista Úbeda y Baeza. I I57: Alfonso VII pierde estos territorios como consecuencia del contrataque almohade.

I 159: Jaén es ocupada por Ibn Hamusk (yerno de Ibn Mardanis). Dominio andalusí independiente hasta 1169.

I 169: Ibn Hamusk reconoce el gobierno almohade, aunque siguió al frente de la ciudad de Jaén.

1225-1226: ocupación de las tierras jiennenses por Fernando III.

1226: conquista definitiva de Baeza.

En algún momento durante este periodo, ante el progresivo avance de las tropas cristianas, la torre de Toya se reestructura profundamente. Esta remodelación consistiría en el levantamiento de un segundo cuerpo de tapial y en la construcción del aljibe, como ya hemos señalado.

En cuanto a la última reforma de la torre (nueva bóveda del aljibe), así como de la muralla no podemos dar una fecha exacta debido a la escasez de información, aunque suponemos, en función del tipo de argamasa, que ambas se realizarían en la misma fecha. De este modo, podemos sugerir una cronología o bien de finales de época almohade, o bien de época cristiana. Las fuentes cristianas nos hablan de la conquista en 1231, por el arzobispo de Toledo, Rodrigo Ximénez de Rada, de Tíscar y Cazorla, junto con otras fortalezas y aldeas de la zona que custodiaban el "paso" del Guadia- 
na Menor, que comunicaba el valle del Guadalquivir, con las Hoyas de Guadix y Baza (QUESADA, 1989). Evidentemente, Toya sería uno de los lugares conquistados, quedando incorporada al adelantamiento de Cazorla. Después de I 310 pasa, junto al territorio de Quesada, a la jurisdicción de Úbeda.

\section{PROBLEMÁTICA ACTUAL DEL ASENTAMIENTO}

En las sucesivas visitas a Toya que a lo largo de este estudio hemos realizado, hemos podido observar la sistemática destrucción que se está llevando a cabo en el Cerro del Castillo y alrededores.

En una primera actuación, se procedió a una reforestación de pinar del 100\% del Cerro del Castillo y aledaños. Los efectos negativos se hicieron palpables inmediatamente, como consecuencia de las remociones de tierra ocasionadas por la pala mecánica con la que se ejecutó la reforestación. Tales remociones conllevaron la alteración del registro arqueológico. Más graves aún serían los efectos que hubiera cabido esperar en el futuro puesto que, con la masiva reforestación de pinar, y debido al particular crecimiento de esta especie, los daños serán plausibles no sólo en el subsuelo sino en las estructuras emergentes todavía hoy. Concretamente, las plantas ocupan el interior de los pequeños bastiones que conforman el lienzo de muralla e incluso podíamos encontrar algunas en contacto con la propia torre.

Unos meses después, se procedió a una nueva actuación en Toya por parte de la Agencia de Medio Ambiente. En este caso, se trata de la ejecución de un amplio cortafuegos que atraviesa el cerro del castillo y lomas contiguas (Lám. 5). Los costes en materia patrimonial son de indudable magnitud puesto que arrasa parte de la Tugia ibero-romana y el lienzo de muralla (la torre, afortunadamente, la rodearon). Resulta cuando menos chocante la política preventiva contra incendios forestales seguida en este caso, puesto que la reforestación de la loma, como era de esperar por las características físicas del medio, no obtuvo éxito alguno.

\section{BIBLIOGRAFÍA}

ABASCAL, J. M.; ESPINOSA, U. (1989): La ciudad hispano-romana: privilegio y poder. Logroño.

ACIÉN, M. (1994): Entre el feudalismo y el Islam. "Umar Ibn Hafsun en los historiadores, en las fuentes y en la historia. Jaén.

ACIÉN, M. (1995): La fortificación en Al-Andalus, La arquitectura militar del Islam Occidental. Madrid.

AGUIRRE, F. J:; JIMÉNEZ, M. C. (1979): Introducción al Jaén islámico. Jaén.

AGUIRRE, F. J:; SALVATIERRA, V. (1989): "Cuando Jaén era Yayyan", Historia de Jaén, vol. II, pp. 453-390. Jaén.

AL-IDRISI (1968): Kitab Nuzhat al-mustaq fi-jtiraq al-afaq. ed. y trad. de R. Dozy y M.J. Goeje, reimpr. Amsterdam.

CASTRO, M.; CHOCLÁN, C. (1988): "El poblamiento rural de la Campiña de Jaén en época imperial", Dedalo, vol. 26, pp. I19-137. Sao Paulo.

DUTRIPON, F.D (1986):: Bibliorum Sacrorum Concordantiae. Hildesheim.

ESLAVA, J.; CEREZO, F.; (1989): Castillos y atalayas del reino de Jaén. Jaén.

FITA, F. (1909): "Fr. Salvador Laín y Rojas. Dos cartas inéditas de este franciscano ilustre", Boletín de la Real Academia de la Historia, LV, cuad. VI, pp. 465-487. Madrid.

GARCÍA MORENO, L. A. (1989): Historia de España Visigoda. Madrid.

GONZÁLEZ, C.; MANGAS, J. (199|): Corpus de Inscripciones Latinas de Andalucía. Jaén. Vol. III. Sevilla.

GUICHARD, P.; CRESSIER, P.; BAZZANA, A. (1988): Les châteaux ruraux d'Al-Andalus. Histoire et archéologie. des husun du sud-est de l'Espagne. Madrid.

GUTIÉRREZ, S. (e. p.): "La ciudad en la Antigüedad Tardía en el Sureste y Levante: la reviviscencia urbana en el marco del conflicto grecogótico", Complutum y las ciudades hispanas en la Antigüedad Tardía (Alcalá de Henares, 1996).

HÜBNER, E. (|87|): Inscriptiones Hispaniae Christianae. Berlín.

HÜBNER, E. (1900): Inscriptionum Hispaniae Christianarum Supplementum. Berlín.

OLMO, L. (1992): "El Reino Visigodo de Toledo y los territorios bizantinos. Datos sobre la heterogeneidad de la Península Ibérica", Coloquio Hispano-Italiano de Arqueología Medieval (Granada, 1990), pp. I85-198. Granada.

PAVÓN, B. (1994): "Arte, arquitectura y arqueología hispanomusulmana (II)", Alqántara, XV, pp. 297-32I.

QUESADA, T. (1989): "La época bajomedieval", Historia de Jaén, vol. II, pp. 49|-519. Jaén. 
SALVADOR, F. (1990):: Hispania Meridional entre Roma y el Islam. Economía y sociedad, Granada.

SALVATIERRA, V. ( 1995): "Continuidad y discontinuidad Romano-Islámica. La campiña de Jaén", Boldrini, E; Francovich, R.: Acculturazione e mutamenti prospettive nell'archeologia medievale del Mediterraneo. Florencia.

\section{RESUMEN:}

En este artículo se estudian las distintas fases constructivas de la Torre de Toya planteándose distintas hipótesis acerca de su cronología. Para ello nos basamos en la prospección de la zona, en la recogida de lo ya publicado acerca de la misma y en el análisis de su contexto histórico.

\section{PALABRAS CLAVE:}

Arquitectura, torre, visigodo, bizantino, inscripción, hisn, muralla, almohade, castellano.
THOMPSON, E. A. (197I): Los godos en España. Madrid. TORRES BALBÁS, L. (197I): Las ciudades hispanomusulmanas. Madrid.

VIVES, J. (1962): Inscripciones cristianas de la España romana y visigoda. Barcelona.

\section{RÉSUMÉ:}

Dans cet article, nous nous proposons d' étudier les différentes phases de contruction de la "Torre de Toya" (Peal de Becerro, Jaén), en portant de quelques hypothèses concernant sa chronologie. Pour ce faire, nous nous sommes fondées sur la prospection de la zone, sur la bibliographie existante à ce sujet et sur I' analyse du contexte historique.

\section{MOTS-CLÉ:}

Architecture, tour, wisigoth, byzantine, inscription, hisn, muraille, almohade, castillan.

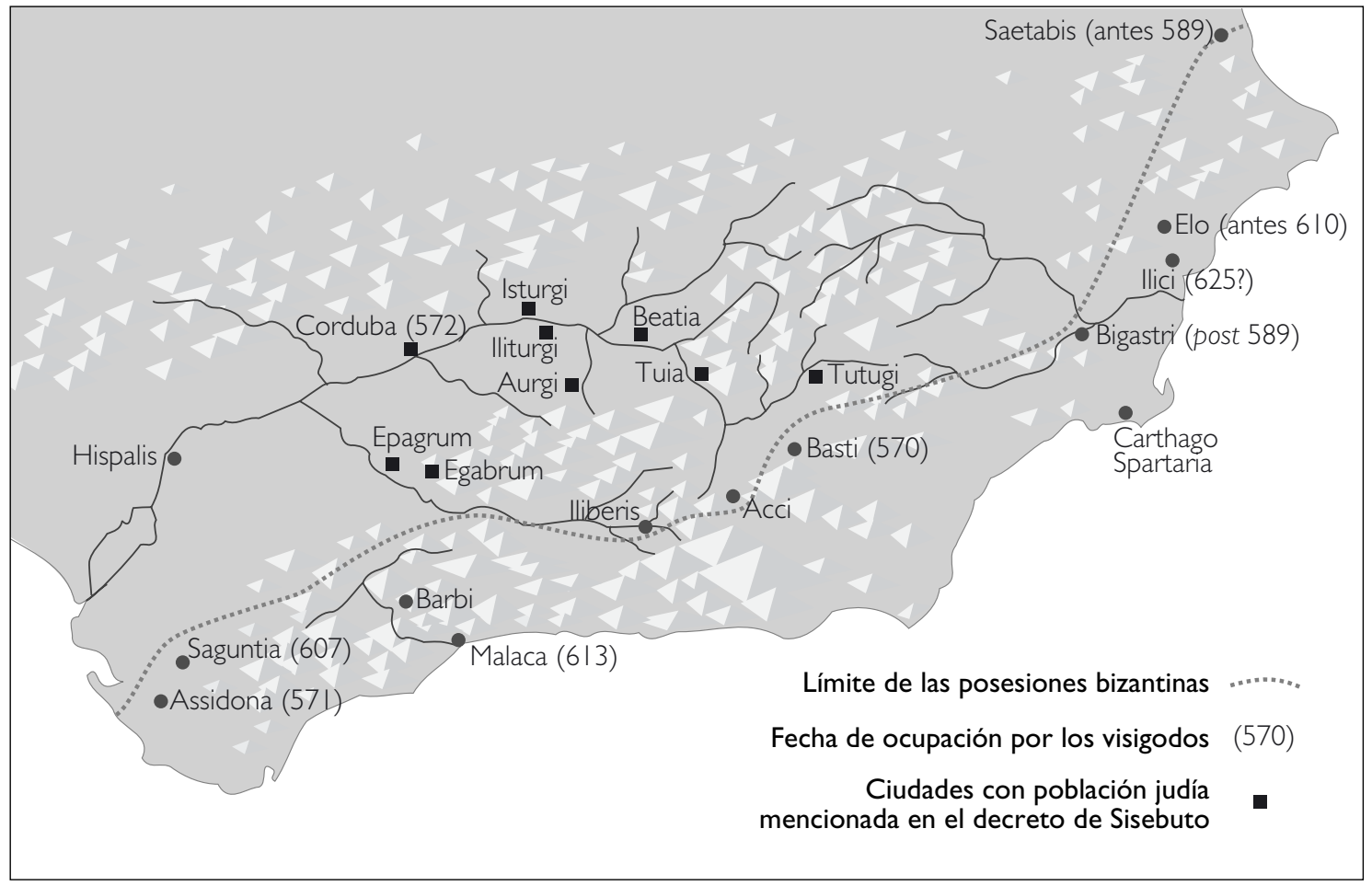

Fig. I. Frontera bizantina y situación de las comunidades judías nombradas en la ley antijudía de Sisebuto. 


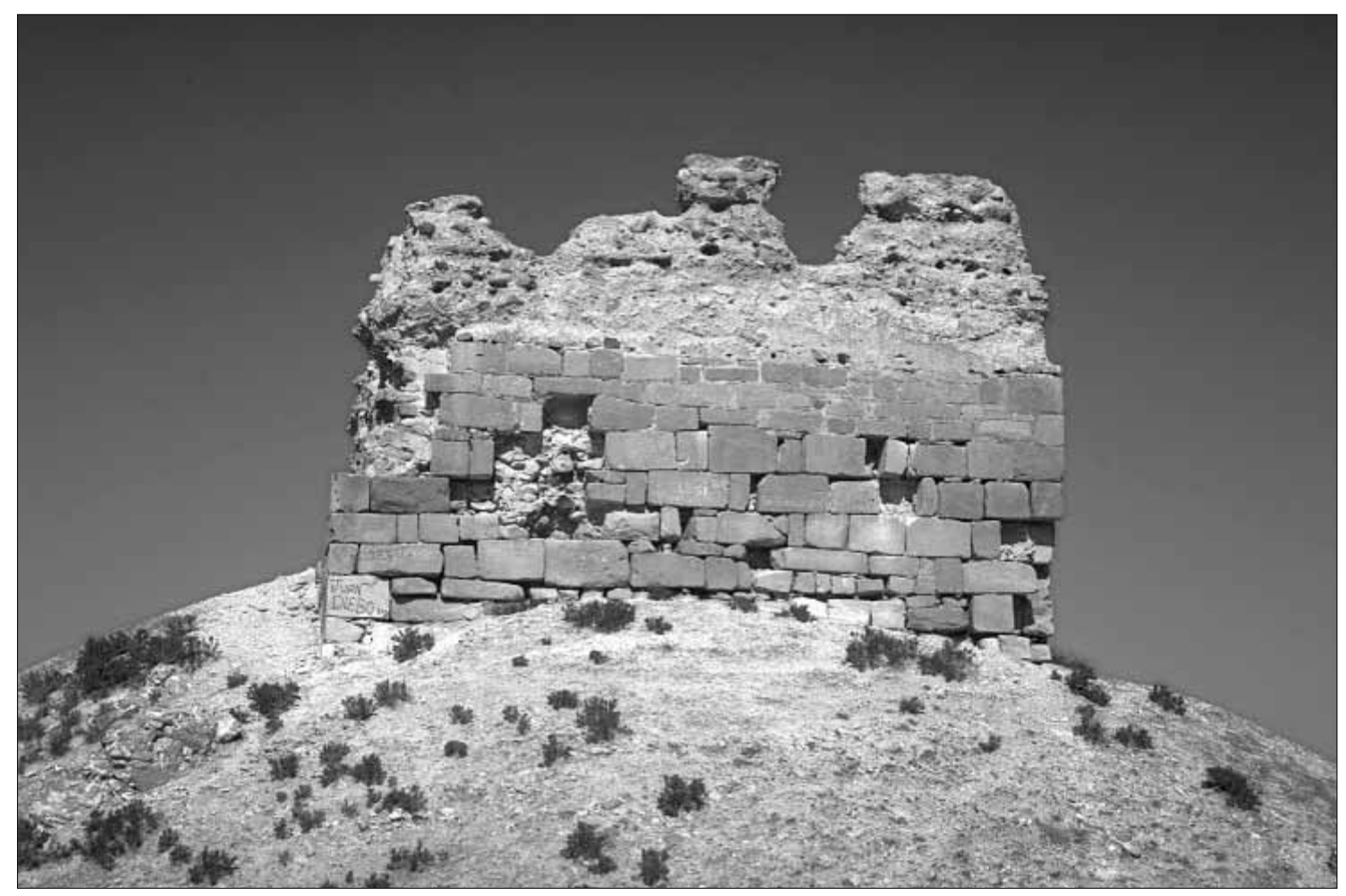

Lám.I. Vista general de la torre de Toya en su cara Sur.

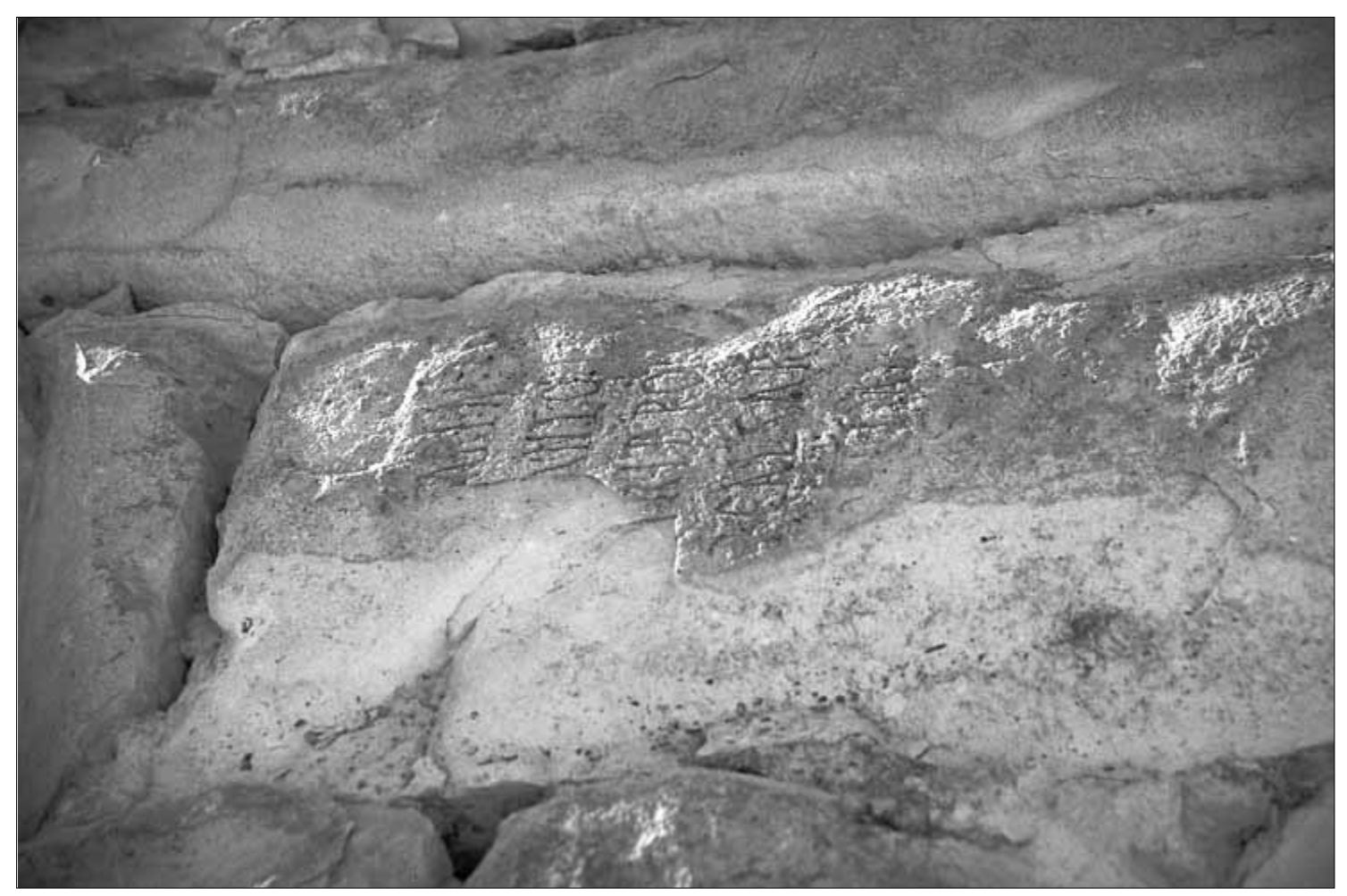

Lám. 2. Detalle de una inscripción utilizada como sillar. 


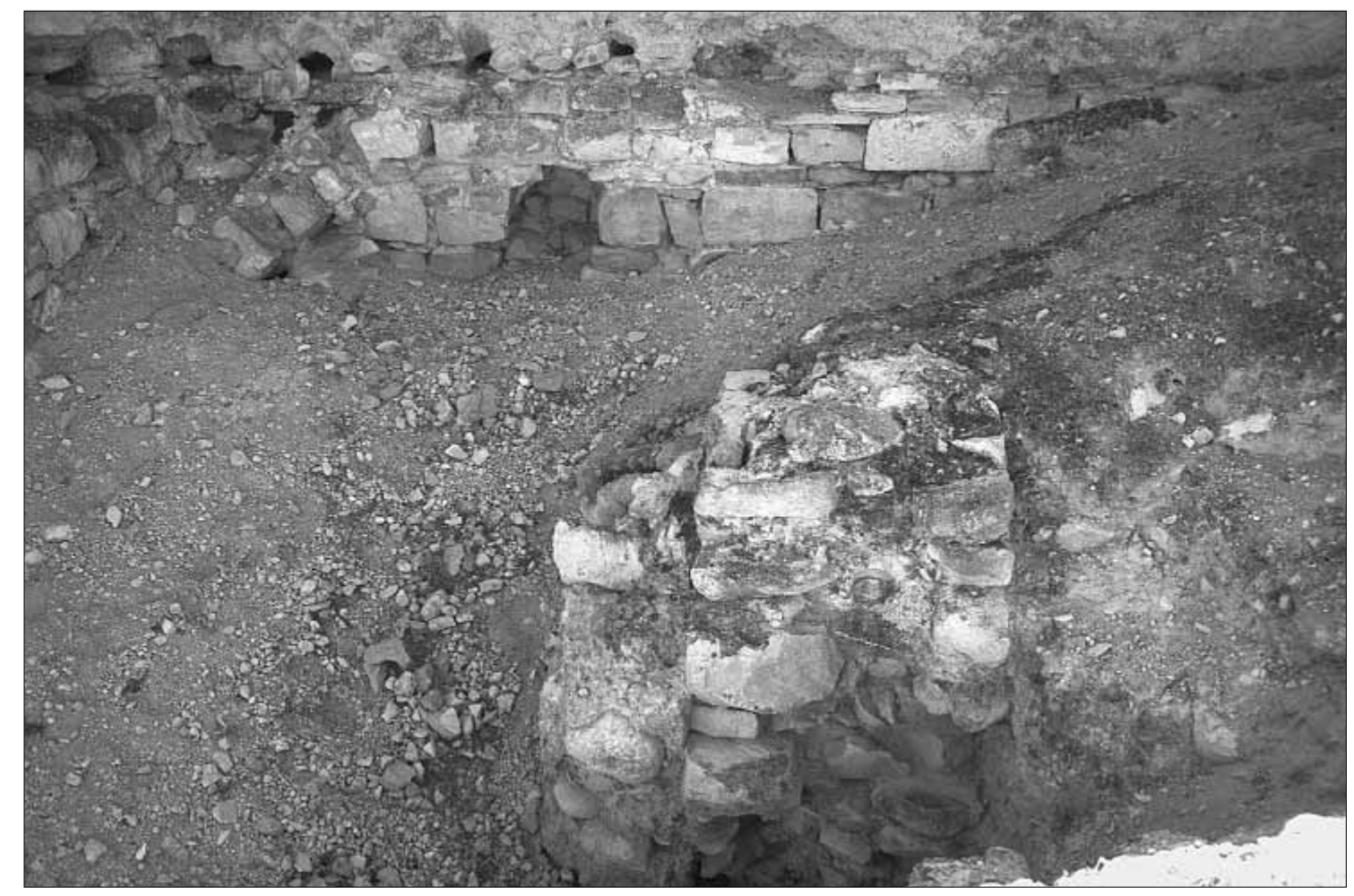

Lám.3. Arranque de la bóveda del aljibe.

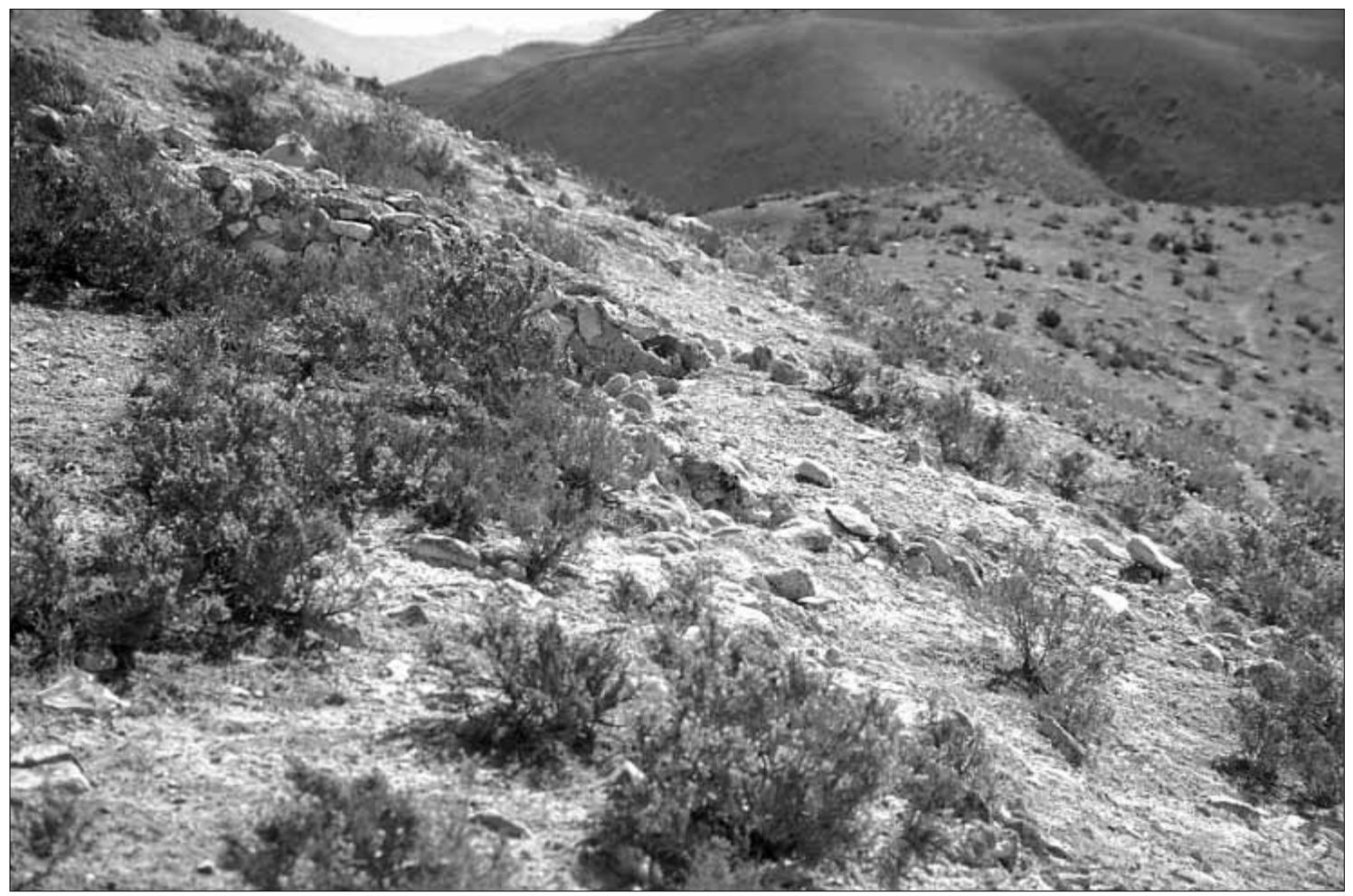

Lám. 4. Vista general del lienzo de muralla. 


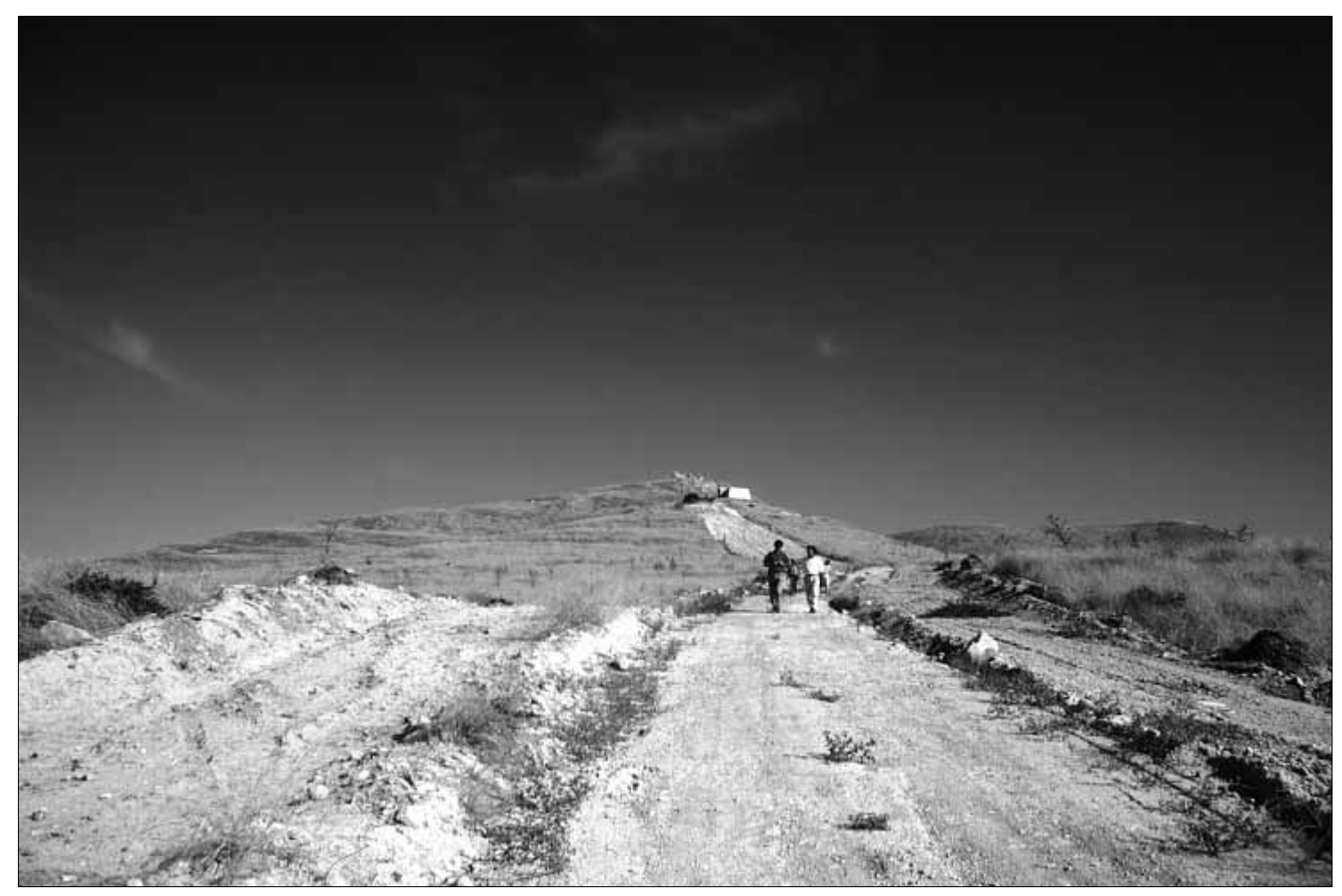

Lám. 5. Vista general del cortafuegos.

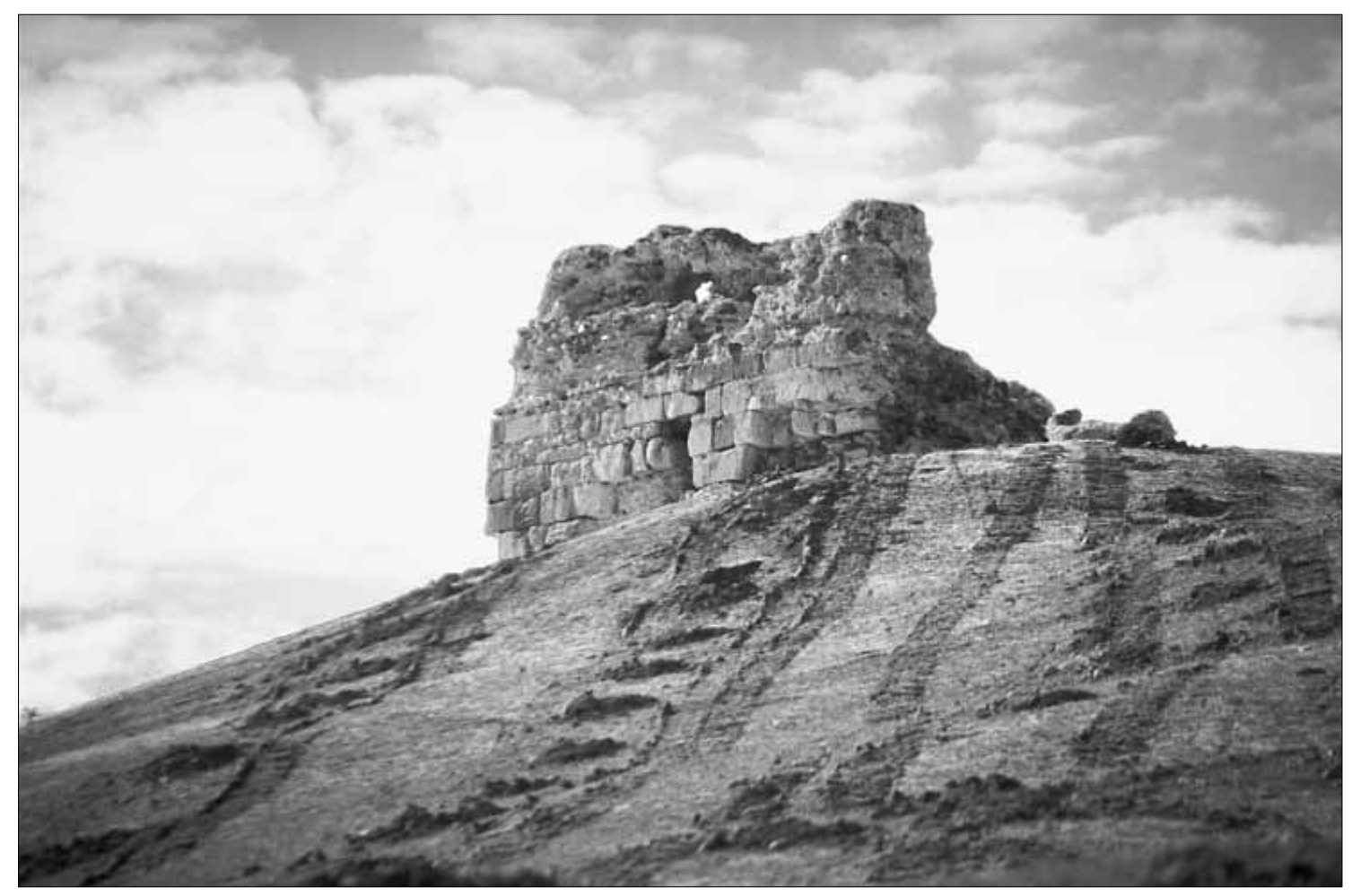

Lám. 6. Detalle del cortafuegos y la torre por el lado sur. 